\title{
Hard X-ray flux from low-mass stars in the Cygnus OB2 association (Research Note)
}

\author{
M. Caramazza ${ }^{1,2}$, J. J. Drake ${ }^{3}$, G. Micela ${ }^{2}$, and E. Flaccomio ${ }^{2}$ \\ 1 Dipartimento di Scienze Fisiche ed Astronomiche, Università di Palermo, via Archirafi 36, 90123 Palermo, Italy \\ e-mail: mcarama@astropa.unipa.it \\ 2 INAF Osservatorio Astronomico di Palermo, Piazza del Parlamento 1, 90134 Palermo, Italy \\ e-mail: [giusi;flaccomio] @astropa.unipa.it \\ 3 Smithsonian Astrophysical Observatory, MS-3, 60 Garden Street, Cambridge, MA 02138, USA \\ e-mail: jdrake@cfa.harvard.edu
}

Received 13 November 2008 / Accepted 29 May 2009

ABSTRACT

\begin{abstract}
Context. The Cygnus OB2 association, the central engine of the Cygnus X star-forming region, is the subject of an extensive INTEGRAL Key Project that will accumulate 6Ms of observations. Analysis of $2 \mathrm{Ms}$ of observations by De Becker and co-workers provides the most sensitive limit yet obtained on hard X-ray emission from the cluster.

Aims. We investigate the X-ray emission in the $20-40 \mathrm{keV}$ band expected from the flaring low-mass stellar population in Cygnus OB2. We discuss whether such emission needs to be considered in the interpretation of existing and future X-ray observations of the region, and whether such observations might provide insight into the high-energy processes on low-mass pre-main sequence stars.

Methods. The total hard X-ray flux from low-mass stars is estimated by assuming the observed soft X-ray emission stems from a superposition of flares. We further assume the ratio of hard X-ray to soft X-ray emission is described by a scaling found for solar flares by Isola and co-workers.

Results. We estimate the low-mass stellar hard X-ray flux in the $20-40 \mathrm{keV}$ band to lie in the range $\sim 2 \times 10^{31}-6 \times 10^{32} \mathrm{erg} \mathrm{s}^{-1}$ and discuss some potential biases that might affect this result.

Conclusions. Hard X-ray emission could lie at a level not much below the current observed flux upper limits for Cygnus OB2. If this emission could be detected, it would provide insight into the hard X-ray production of large flares on pre-main sequence stars. We highlight the penetrating power of hard X-rays from low-mass stellar populations as a possible pointer to our Galaxy's hidden star-forming clusters and super-clusters using more sensitive observations from future missions.
\end{abstract}

Key words. stars: coronae - stars: flare - Galaxy: open clusters and associations: individual: Cygnus OB2

\section{Introduction}

Motivated by the detection of nonthermal radio emission from early-type stars in the nearby massive Cygnus OB2 association $(d=1.7 \mathrm{kpc}$, Massey \& Thompson 1991), De Becker et al. (2007) have recently used an extensive set of observations obtained by the International Gamma-Ray Laboratory (INTEGRAL) IBIS instrument to search for hard X-ray emission from some of the more prominent radio sources. The nonthermal radio emission from these stars is thought to arise from a relativistic population of electrons accelerated by diffusive shock acceleration in their interacting stellar winds (e.g. Pittard \& Dougherty 2006). De Becker et al. (2007) note that hard X-rays might plausibly be produced by inverse Compton scattering of photospheric UV photons.

One other potential source of hard X-rays in Cygnus OB2 are the myriad flares thought to arise from magnetic reconnection events that characterize and sustain the coronae of the low-mass stellar population (see, e.g., Albacete Colombo et al. 2007a; Caramazza et al. 2007). The flare paradigm developed from the solar perspective posits that hard X-rays can arise from the impact on dense photospheric gas of electrons accelerated to mildly relativistic energies (the "thick-target model", e.g. Brown 1971; Hudson 1972; Lin \& Hudson 1976). Tentative direct evidence for such nonthermal hard X-rays up to $200 \mathrm{keV}$ has recently been found from Swift observations of a large flare on the RS CVnlike interacting binary II Peg Osten et al. (2007). Large stellar flares observed on the young single star AB Dor and the active binaries Algol and UX Ari by the BeppoSAX mission (Boella et al. 1997) in the $0.1-100 \mathrm{keV}$ range revealed evidence of similarly energetic emission, though it was not possible to ascertain whether this was of thermal or nonthermal origin (Maggio et al. 2000; Favata \& Schmitt 1999; Franciosini et al. 2001).

While any single flare event on a low-mass star is a trifling insignificance in comparison with the effusive vigor of its highmass brethren, the comparative multitude of the former population in Cygnus OB2 might render a palpable combined effect. An estimate of this effect is motivated from different standpoints.

Firstly, the search for hard X-rays from early-type stars by De Becker et al. (2007) resulted in only upper limits; an estimate of the flux limit at which low-mass stars might be detected could be important for understanding the origin of any hard X-rays detected in deeper observations by INTEGRAL or future missions.

Secondly, the large concentration of low-mass stars in the Cygnus OB2 region might also provide insight into the highenergy nature of stellar flares that is difficult to attain from observations of nearby single stars that rarely exhibit large events. 
Isola et al. (2007) have recently shown that a well-defined power-law relationship between soft and hard X-rays seen in solar flares matches the $20-40 \mathrm{keV}$ flux observed during the AB Dor, Algol and UX Ari flares, but tends to overpredict the, presumed nonthermal, $60-80 \mathrm{keV}$ flux. Alexander \& Daou (2007) find evidence for a saturation in nonthermal X-ray flux above $20 \mathrm{keV}$ for solar flares with increasing soft X-ray luminosity, and it is conceivable that this is related to the deficit in observed nonthermal stellar X-rays. Detection of, or limits on, the hard X-ray flux from the low-mass Cygnus OB2 population could provide constraints for extrapolating solar flare models to much more active pre-main sequence stars.

Thirdly, the hard X-ray environment of young protostellar systems is potentially relevant for the ionization of circumstellar material and protoplanetary disks (e.g. Glassgold et al. 1997; Aikawa \& Herbst 1999; Fromang et al. 2002; Matsumura \& Pudritz 2003). We currently have no information on the intensity of this radiation environment.

We outline in Sect. 2 below a calculation of the hard X-ray flux from the Cygnus OB2 low-mass population under the assumption that the observed soft X-rays from these stars is dominated by a superposition of flaring events. We then discuss in Sect. 3 the uncertainties in this estimate, the degree to which it likely overestimates the true level of hard X-ray emission, and the implications of the results.

\section{Estimating the low-mass stellar hard X-ray flux}

There are three main components to our hard X-ray flux estimate: the Cygnus OB2 initial mass function (IMF) and total stellar mass; the relationship between stellar mass and observed soft X-ray luminosity for the low-mass population; and the relationship between the soft and hard X-ray luminosities. In order to gain a better perspective on the uncertainties involved in our estimate, we use three different approaches that employ these ingredients in slightly different ways.

We adopt the IMF derived by Knödlseder (2000) based on 2MASS photometry. That study noted the power-law index found, $\Gamma=-1.6 \pm 0.1$, is compatible with that of Kroupa et al. (1993) for the solar neighborhood, but is significantly steeper than found from spectroscopy of the central region of Cygnus OB2 by Massey \& Thompson (1991). We therefore also investigate below the effect of a different power-law slope, and in particular that found for the Orion Nebula Cluster (ONC, $\Gamma=-1.2$ ) by Muench et al. (2002). We consider hard X-rays from stars in the mass range $0.3-2 M_{\odot}$; the upper limit corresponds to the approximate boundary between low-mass premain sequence stars with outer convection zones and intermediate mass stars that should have radiative envelopes; the lower mass limit corresponds to the limit of the Knödlseder (2000) study - essentially our limit of current knowledge. For stars with mass $\lesssim 0.3 M_{\odot}$, X-ray luminosities of T Tauri stars are observed to decline much more rapidly with decreasing mass than for higher mass stars (e.g. Preibisch et al. 2005; Albacete Colombo et al. 2007b), rendering their contribution to the total X-ray luminosity less significant, regardless of whether the IMF flattens toward lower masses or not (e.g. Scalo 1986; Kroupa et al. 1993; Kroupa 2001; Chabrier 2003). Nevertheless, we also investigate the effects of this lower mass cut-off in our calculations below.

Albacete Colombo et al. (2007a) found the X-ray luminosities of low-mass stars in Cygnus OB2 to be essentially identical to those in the ONC (Preibisch et al. 2005). We therefore adopt the relation between stellar mass, $M$, and X-ray luminosity, $L_{\mathrm{X}}$, from the ONC study. In order to examine the effects on our calculation of the significant scatter in observed $L_{\mathrm{X}}$ vs. $M$, we also calculate the expected hard X-ray flux using the observed ONC stellar sample renormalized to the Cygnus OB2 low-mass content (see Sect. 2.3)

The relationship between the soft and hard X-ray luminosities was derived using the extrapolation of the relation found for solar flares by Isola et al. (2007), $F_{20-40}=9.9 \times 10^{6} F_{\mathrm{G}}^{1.37}$, where $F_{20-40}$ is the $20-40 \mathrm{keV}$ flux density in $\mathrm{ph} \mathrm{cm}^{-2} \mathrm{keV}^{-1} \mathrm{~s}^{-1}$ and $F_{\mathrm{G}}$ is the Geostationary Operational Environment Satellite (GOES) $1.6-12.4 \mathrm{keV}$ flux in unit of $\mathrm{W} \mathrm{m}^{-2}$. In order to convert this relation to the stellar case, we assumed that the observed soft X-ray luminosities of the Cygnus OB2 low-mass premain sequence population could be explained by a superposition of flares. This assumption is based on evidence that the solar corona is largely characterized by a power-law flare distribution in total energy, $E$, and frequency, $N, \mathrm{~d} N / \mathrm{d} E \propto E^{-\alpha}$ (e.g. Lin et al. 1984; Krucker \& Benz 1998; Hudson 1991), and on studies of EUV and X-ray flares and photon arrival times for active stars that suggest a similar flare distribution with $\alpha \sim 2$ (Audard et al. 2000; Kashyap et al. 2002; Güdel et al. 2003). The highenergy tails of flares detected in Chandra studies of the ONC and Cygnus OB2 are also well-approximated by a power-law frequency distribution with $\alpha=2.2 \pm 0.2$ (ONC, Caramazza et al. 2007), $\alpha=2.1 \pm 0.1$ (Cygnus OB2, Albacete Colombo et al. 2007a). In the following we outline our estimates, and then discuss the uncertainties of this approach. An alternative approach to estimate the nonthermal emission is proposed in (Güdel 2009).

\subsection{Hard-soft $X$-ray luminosity relation for young low-mass stars}

The relation from Isola et al. (2007) relates the flux in the 1.6$12.4 \mathrm{keV}$ band to the flux density in the $20-40 \mathrm{keV}$ band. In order to obtain a relation between the peak luminosity of flares in the two bands, we first converted the GOES flux from $\mathrm{W} \mathrm{m}^{-2}$ into $\mathrm{erg} \mathrm{s}^{-1}$ luminosity, and the flux density in the hard band, given in unit of $\mathrm{ph} \mathrm{cm}^{-2} \mathrm{keV}^{-1} \mathrm{~s}^{-1}$, into erg s${ }^{-1}$ luminosity, assuming a photon energy of $20 \mathrm{keV}$ and multiplying for the band range. We also converted the $1.6-12.4 \mathrm{keV}$ luminosity into luminosity in the $0.5-8.0 \mathrm{keV}$ band: the conversion factor $\left(K_{\mathrm{conv}}=0.34\right)$ was evaluated considering a Raymond Smith model with $k T=$ $1.35 \mathrm{keV}$ and $\log \left(N_{\mathrm{H}}\right)=22.25 \mathrm{~cm}^{-2}$ (Albacete Colombo et al. 2007b). We then obtain the following relation:

$\mathcal{L}_{f l(20-40)}=1.8 \times 10^{28} \cdot\left(1.2 \times 10^{-31}\right)^{\beta} \cdot\left(\mathcal{L}_{f l(0.5-8.0)}\right)^{\beta} \operatorname{erg~s}^{-1}(1)$

where $\beta=1.37 \pm 0.07$ is the index found by Isola et al. (2007). Note that the factor in the previous relation varies strongly due to the uncertainty of $\beta$.

This relation can be converted in a relation between the total energy output of flares in the two spectral bands, considering that $E=\mathcal{L}_{f l} \cdot \tau$. Given the power-law distribution of flare energies in the $0.5-8.0 \mathrm{keV}$ band, we conclude that the $20-40 \mathrm{keV}$ band flare energies are also distributed as a power-law. The index of this power-law, $\gamma$, is a function of $\alpha$ and $\beta: \gamma=(\alpha+\beta-1) / \beta$, where $2.0 \leq \alpha \leq 2.4$ (Caramazza et al. 2007) and $1.30 \leq \beta \leq 1.44$ (Isola et al. 2007). If we impose the condition that $\gamma$ also has a value greater than 2 , i.e. that the $20-40 \mathrm{keV}$ band emission is due entirely to flares, then the acceptable values for $\alpha$ and $\beta$ fall in the ranges $1.31<\beta<1.39$ and $2.32<\alpha<2.4$. Integrating the distribution of flares between the minimum energy and the maximum energy, that we can set as infinite, we obtain the 
following relation between stellar luminosity in the $20-40 \mathrm{keV}$ and $0.5-8.0 \mathrm{keV}$ bands:

$L_{20-40}=A \cdot L_{0.5-8.0} \mathrm{erg} \mathrm{s}^{-1}$

where

$A=\frac{\tau_{20-40}}{\tau_{0.5-8.0}^{\beta}} \frac{(\alpha-2) \cdot E_{0.5-8.0 \min }^{\beta-1}}{\alpha-\beta-1} 1.8 \times 10^{28} \cdot\left(3.5 \times 10^{-31}\right)^{\beta}$

here, we set $\tau_{0.5-8.0}$ to $10 \mathrm{~h}$ (Caramazza et al. 2007), while $\tau_{20-40}$ is scaled using the ratio $(0.16)$ derived from the median duration of flares in the hard and soft bands calculated by Veronig et al. (2002) from a sample of solar flares. $E_{0.5-8.0}$ min was set to $\sim 2 \times 10^{32}$ erg that is the median values obtained from a statistical analysis of the ONC lightcurves interpreted assuming the emission enterely due to flares with a power law distribution (Caramazza et al. 2007). The energy of flares that contribute to the star emission in Caramazza et al. (2007) is given in counts. We converted the counts to energy, assuming a Raymond-Smith emission with the median values of $N_{\mathrm{H}}$ and $k T$ of the ONC sample $\left(N_{\mathrm{H}}=1.9 \times 10^{21} \mathrm{~cm}^{-2} k T=1.25 \mathrm{keV}\right)$. Note that, since $\beta$ is not too different from 1 , the hard $\mathrm{X}$-ray luminosity is relatively insensitive to change in $E_{0.5-8.0}$ min or $\tau_{0.5-8.0}$ values: a factor of 10 change in either of these quantities results in only a factor of 2 difference in A. Assuming uniform distributions of $\alpha$ and $\beta$ in the total intervals given above, we estimate that $90 \%$ of the resulting values for the proportionality factor $\mathrm{A}$ lie in the range $A=\left[1.8 \times 10^{-4}, 3.0 \times 10^{-3}\right]$. Allowing also for significant uncertainties in the other parameters $\left(\tau_{20-40} / \tau_{0.5-8.0}=[0.08,0.24]\right.$, $\left.\tau_{0.5-8.0}=[3,18] \mathrm{h}, E_{0.5-8.0 \min }=\left[2 \times 10^{31}, 2 \times 10^{33}\right] \mathrm{erg}\right)$, the $90 \%$ interval for $A$ becomes $\left[1.1 \times 10^{-4}, 4.0 \times 10^{-3}\right]$. We will adopt in the following the latter uncertainty range.

\subsection{Analytical estimate}

Adopting the relation between stellar mass and the X-ray luminosity found for the Chandra Orion Ultra-deep Project (COUP) stars in the range $0.3-2 M_{\odot}$ (Preibisch et al. 2005),

$\log \left(L_{0.5-8.0}\right)=30.37+1.44 \cdot \log (M)$

and combining this with Eq. (2), we obtain a relation between stellar mass and hard X-ray flux. This expression can then be integrated over the stellar mass distribution for Cygnus OB2. The IMF found by Knödlseder (2000) follows the standard form

$\frac{\mathrm{d} N}{\mathrm{~d} M}=k M^{\Gamma-1}=k \cdot M^{-2.6} \quad$ with $\Gamma=-1.6$,

and assuming for the present a total mass of $M_{\mathrm{tot}}=5 \times 10^{4} M_{\odot}$ (Knödlseder 2000), and maximum and minimum stellar masses in the cluster of $M_{\max }=80 M_{\odot}$ and $M_{\min }=0.3 M_{\odot}$, respectively, the normalization constant is $k=1.5 \times 10^{4}$.

In order to calculate the total hard X-ray luminosity in the cluster, we transform the IMF into a function of $20-40 \mathrm{keV}$ stellar X-ray luminosity, and integrate between the luminosities corresponding to 0.3 and $2.0 M_{\odot}$, obtaining values in the following range:

$L_{20-40}^{\mathrm{Cyg}}=\left[1.8 \times 10^{31}, 6.6 \times 10^{32}\right] \mathrm{erg} \mathrm{s}^{-1}$.

\subsection{Scaling the Orion hard $X$-ray luminosity}

We can also make a rough estimate of hard X-ray flux from Cygnus OB2 by scaling directly the observed X-ray luminosity distribution of the low-mass stars in Orion. This approach has the advantage that the observed scatter in the COUP sample is intrinsically included. The IMF of the Orion sample has a shallower slope than that for Cygnus OB2 of Knödlseder (2000), with $\Gamma=-1.2$ (Muench et al. 2002), and we investigate the influence of this difference in Sect. 2.4 below.

The total 20-40 keV luminosity for the COUP sample is simply the sum over all stars of mass $M_{i}$ in the range $0.3-2 M_{\odot}$,

$L_{20-40}^{\mathrm{COUP}}=A \cdot \sum_{M_{i}=0.3}^{2} L_{0.5-8.0}(i)=\left[8.6 \times 10^{28}, 3.1 \times 10^{30}\right] \mathrm{erg} \mathrm{s}^{-1}$,

where the relation between the $20-40 \mathrm{keV}$ and $0.5-8 \mathrm{keV}$ luminosities is given by Eq. (2). Assuming a similar IMF for both Orion and Cygnus OB2, the hard X-ray luminosity of the latter is then simply given by the product of $L_{20-40}^{\text {COUP }}$ and the ratio of cluster masses within the $0.3-2 M_{\odot}$ mass interval. The Cygnus OB2 IMF from Eq. (5) yields a total mass in this range of $M_{0.3-2}^{\text {Cyg }}=3.5 \times 10^{4} M_{\odot}$, while the analogous total mass of the COUP sample is $M_{0.3-2}^{\text {COUP }}=175 M_{\odot}$. Even in this case the total Cygnus OB2 hard X-ray luminosity is therefore

$L_{20-40}^{\mathrm{Cyg}}=\left[1.7 \times 10^{31}, 6.3 \times 10^{32}\right] \mathrm{erg} \mathrm{s}^{-1}$.

\subsection{Simulating Cygnus OB2 using the Orion sample}

In order to account for the different IMF slopes in Cygnus OB2 and Orion $(\Gamma=-1.6 \mathrm{cf}$. -1.2$)$, we also performed a simple Monte Carlo simulation of 10000 stars. The mass range $0.3-2 M_{\odot}$ was divided into 10 bins, and within each mass bin 0.5-8.0 keV luminosities were randomly selected from those observed in the COUP sample. The number of "stars" drawn in each bin was weighted according to the Cygnus OB2 IMF from Eq. (5), and the luminosity for each star was scaled to $L_{20-40}$ as described earlier. The luminosity of the simulated sample was then scaled so as to have a total mass equal to that of Cygnus OB2 for the $0.3-2 M_{\odot}$ mass range. In this way, we estimate

$L_{20-40}^{\mathrm{Cyg}}=\left[1.9 \times 10^{31}, 6.6 \times 10^{32}\right] \mathrm{erg} \mathrm{s}^{-1}$.

As a verification of the calculation, we recovered a luminosity of $L_{20-40}^{\text {COUP }}=\left[8.9 \times 10^{28}, 3.2 \times 10^{30}\right] \mathrm{erg} \mathrm{s}^{-1}$ - essentially the same as that found from direct calculation in Sect. 2.3 - for an IMF with slope $\Gamma=-1.2$ scaled to the appropriate COUP total mass.

\section{Discussion}

The range of 20-40 keV fluxes we have evaluated above can be compared with the the $3 \sigma 20-60 \mathrm{keV}$ flux upper limit of $6.1 \times 10^{-12} \mathrm{erg} \mathrm{cm} \mathrm{cm}^{2} \mathrm{~s}^{-1}$ obtained from 2.12 Ms of INTEGRAL IBIS-ISGRI observations by De Becker et al. (2007) for the unidentified $\gamma$-ray source $3 E G$ J20033 +4188 that lies in the close vicinity of Cygnus OB2 ${ }^{1}$. Adopting a distance of $1.7 \mathrm{kpc}$ for Cygnus OB2 (e.g. Massey \& Thompson 1991), this flux corresponds to a luminosity $\sim 2 \times 10^{33} \mathrm{erg} \mathrm{s}^{-1}$.

\footnotetext{
1 The distance between the position of 3EG J20033+4188 and the center of Cyg OB2 obtained by (Knödlseder 2000) is 7'.
} 
The 20-40 keV bandpass considered here is significantly narrower that the 20-60 keV range cited by De Becker et al. (2007). Based on the power-law spectrum and slope estimate by Isola et al. (2007), we expect the corresponding estimate for the 20-60 keV range to be a factor 1.8 higher. Moreover, the upper limit of the fluxes for the undetected stars was estimated by De Becker et al. (2007) within a PSF of 12'. Using the King profile describing the projected spatial distribution of starlight from the association found by Knödlseder (2000), the ratio between the flux in the whole association area (radius $\sim 60^{\prime}$ ) to that in the 3 EG J20033+4188 region is 6.6. Applying these scaling factors, the total luminosity in the whole region of Cygnus should not be higher than $7.3 \times 10^{33} \mathrm{erg} \mathrm{s}^{-1}$. While this scaling is somewhat crude, the range of $20-40 \mathrm{keV}$ fluxes we have evaluated is one order of magnitude lower than this upper limit. This confirms the potential importance of hard X-ray emission from the low-mass stars in stellar clusters.

There are several assumptions that might have led us to overestimate the Cygnus OB2 luminosity. First, the total cluster mass of (4-10) $\times 10^{4} M_{\odot}$ derived by Knödlseder (2000) has been challenged by Hanson (2003), who estimated the cluster to lie slightly closer at $1.4 \mathrm{kpc}$ and suggested a total cluster mass closer to $10^{4} M_{\odot}$. Nevertheless, based on a study of A-stars in the Cygnus OB2 field, Drew et al. (2008) find that "a total mass of 30000-40000 $M_{\odot}$ would not be surprising". Our adopted $5 \times 10^{4} M_{\odot}$ is then perhaps up to $1.2-1.7$ times too high.

We have also assumed that all of the observed stellar X-ray flux is due to continuous flaring. Some fraction of the observed luminosity of these stars might also be attributed to a quiescent component.

One additional factor of uncertainty is related to the duration of flares. Indeed, the hard X-rays seen in solar flares that are usually associated with the flare impulsive phase generally decay on a more rapid timescale than soft X-rays (e.g. Dennis \& Zarro 1993; Benz 2008, and references therein). We considered a ratio between the duration of flares in hard and soft band derived from solar measures of flare duration (Veronig et al. 2002). The case of large stellar flares is not so clear, however. The decay of the $14-40 \mathrm{keV}$ flux in the large II Peg flare observed by Swift was not obviously shorter than that for the soft X-rays. It should also be noted that in large stellar flares there will be a larger thermal contribution in the $20-40 \mathrm{keV}$ range than in much less energetic solar flares, in alignment with the general correlation of increasing plasma temperature with flare total energy (e.g. Feldman et al. 1995). Moreover, also the minimum energy of the distribution of flares is uncertain, we set it to the median value obtained from the analysis of the COUP low mass samples. In our estimation we considered that it can be uncertain of two orders of magnitude, that implies a variation of a factor $\sim 5$ in the resulting total energy: note, however, that Güdel (2009) found a value even lower (few times $10^{30}$ ).

Our assumptions could lead to an overestimation of the real value of the total luminosity, but a value of $\sim 6 \times 10^{32} \mathrm{erg} \mathrm{s}^{-1}$, an order of magnitude below the De Becker et al. (2007) flux upper limit, would render the low-mass population of importance in searches for emission from specific suspected sources of hard $\mathrm{X}$-rays, such as the unidentified $\gamma$-ray source 3EG $2033+4118$, the unidentified $\mathrm{TeV}$ source $\mathrm{TeV} \mathrm{J} 2032+4130$, and massive colliding-wind binaries.

The possibility of detecting hard X-ray emission from nearby clustered low-mass pre-main sequence populations provides a promising means of investigating high-energy processes on stars that are generally too distant to study in detail individually. Of particular interest is the relation between soft and hard X-ray emission of solar flares (e.g. Isola et al. 2007; Battaglia et al. 2005), and how the underlying physical basis can be extrapolated to the other regimes, such as the pre-main sequence stellar case where hard X-rays from flares can be important agents of protoplanetary disk ionisation (e.g. Igea \& Glassgold 1999; Glassgold et al. 2004).

Our discussion assumes that all stars will be "observed" in the $20-40 \mathrm{keV}$ range, i.e., that X-rays will penetrate both the circumstellar and ambient cluster and line-of-sight extinction. Cygnus OB2 is located behind the Great Cygnus Rift that leads to extinction of up to (and possibly beyond) $A_{\mathrm{V}} \sim 10$ (e.g. Massey \& Thompson 1991). Extinction of $10^{m}$ corresponds to a neutral hydrogen column density $N_{\mathrm{H}} \sim 1.5 \times 10^{22} \mathrm{~cm}^{-2}$. The optical depth for such a column for $20-40 \mathrm{keV}$ X-rays is completely negligible; the Compton ionization and scattering crosssection of $\sigma_{T} \sim 6.65 \times 10^{-25} \mathrm{~cm}^{2}$ renders the interstellar medium optically thin to hard X-rays for columns up to $N_{\mathrm{H}} \sim 10^{24} \mathrm{~cm}^{-2}$. Except perhaps in rare cases of stars obscured by very dense circumstellar disk mid-plane gas and dust, the assumption that we will see the whole cluster in hard X-rays should be valid.

Finally, this consideration raises the possibility of using the penetrating power of hard X-rays as a pointer to our Galaxy's hidden superclusters. Hanson (2003) noted that extrapolation of the locally-derived Galactic cluster luminosity function indicates that our Galaxy hosts "tens to perhaps a hundred" massive clusters with total mass $\sim 10^{4} M_{\odot}$. These clusters are likely hidden behind many magnitudes of extinction and will not be easy to locate. Hard X-rays can penetrate such extinction, and the arrival of missions in the next decade able to provide relatively precise imaging with much greater sensitivity in the $\sim 10-100 \mathrm{keV}$ bandpass, such as NUSTAR (Harrison et al. 2005) and Symbol-X (Pareschi \& Ferrando 2005), could provide an assay of this hitherto much overlooked population of our Galaxy. In this context, Cygnus OB2 represents a potential "Rosetta Stone", offering a nearby super-cluster example that can be well-characterized using multi-wavelength techniques that will not be applicable to more distant and extinguished clusters.

Acknowledgements. J.J.D. was funded by NASA contract NAS8-39073 to the Chandra X-ray Center (CXC) during the course of this research and thanks the CXC director, Harvey Tananbaum, and the CXC science team for advice and support. J.J.D. also thanks the ISHERPA program for financial support during his visit to the Osservatorio Astronomico di Palermo, and the Osservatorio director, Prof. S. Sciortino, and staff for their help and warm hospitality. M.C., G.M. and E.F. acknowledge financial support from the Ministero dell'Università e della Ricerca and ASI/INAF Contract I/023/05/0. J.J.D. thanks Jürgen Knödlseder for useful discussions that partly inspired this work.

The authors thank the referee Manuel Güdel for suggestions and useful comments that improved this work.

\section{References}

Aikawa, Y., \& Herbst, E. 1999, A\&A, 351, 233

Albacete Colombo, J. F., Caramazza, M., Flaccomio, E., Micela, G., \& Sciortino, S. 2007a, A\&A, 474, 495

Albacete Colombo, J. F., Flaccomio, E., Micela, G., Sciortino, S., \& Damiani, F. 2007b, A\&A, 464, 211

Alexander, D., \& Daou, A. G. 2007, ApJ, 666, 1268

Audard, M., Güdel, M., Drake, J. J., \& Kashyap, V. L. 2000, ApJ, 541, 396

Battaglia, M., Grigis, P. C., \& Benz, A. O. 2005, A\&A, 439, 737

Benz, A. O. 2008, Living Reviews in Solar Physics, 5, 1

Boella, G., Butler, R. C., Perola, G. C., et al. 1997, A\&AS, 122, 299

Brown, J. C. 1971, Sol. Phys., 18, 489

Caramazza, M., Flaccomio, E., Micela, G., et al. 2007, A\&A, 471, 645

Chabrier, G. 2003, PASP, 115, 763

De Becker, M., Rauw, G., Pittard, J. M., et al. 2007, A\&A, 472, 905

Dennis, B. R., \& Zarro, D. M. 1993, Sol. Phys., 146, 177

Drew, J. E., Greimel, R., Irwin, M. J., \& Sale, S. E. 2008, MNRAS, 521

Favata, F., \& Schmitt, J. H. M. M. 1999, A\&A, 350, 900 
Feldman, U., Laming, J. M., \& Doschek, G. A. 1995, ApJ, 451, L79 Franciosini, E., Pallavicini, R., \& Tagliaferri, G. 2001, A\&A, 375, 196 Fromang, S., Terquem, C., \& Balbus, S. A. 2002, MNRAS, 329, 18

Glassgold, A. E., Najita, J., \& Igea, J. 1997, ApJ, 480, 344

Glassgold, A. E., Najita, J., \& Igea, J. 2004, ApJ, 615, 972

Güdel, M. 2009, ArXiv e-prints

Güdel, M., Audard, M., Kashyap, V. L., Drake, J. J., \& Guinan, E. F. 2003, ApJ, 582,423

Hanson, M. M. 2003, ApJ, 597, 957

Harrison, F. A., Christensen, F. E., Craig, W., et al. 2005, Exper. Astron., 20, 131

Hudson, H. S. 1972, Sol. Phys., 24, 414

Hudson, H. S. 1991, Sol. Phys., 133, 357

Igea, J., \& Glassgold, A. E. 1999, ApJ, 518, 848

Isola, C., Favata, F., Micela, G., \& Hudson, H. S. 2007, A\&A, 472, 261

Kashyap, V. L., Drake, J. J., Güdel, M., \& Audard, M. 2002, ApJ, 580, 1118

Knödlseder, J. 2000, A\&A, 360, 539
Kroupa, P. 2001, MNRAS, 322, 231

Kroupa, P., Tout, C. A., \& Gilmore, G. 1993, MNRAS, 262, 545

Krucker, S., \& Benz, A. O. 1998, ApJ, 501, L213

Lin, R. P., \& Hudson, H. S. 1976, Sol. Phys., 50, 153

Lin, R. P., Schwartz, R. A., Kane, S. R., Pelling, R. M., \& Hurley, K. C. 1984, ApJ, 283, 421

Maggio, A., Pallavicini, R., Reale, F., \& Tagliaferri, G. 2000, A\&A, 356, 627

Massey, P., \& Thompson, A. B. 1991, AJ, 101, 1408

Matsumura, S., \& Pudritz, R. E. 2003, ApJ, 598, 645

Muench, A. A., Lada, E. A., Lada, C. J., \& Alves, J. 2002, ApJ, 573, 366

Osten, R. A., Drake, S., Tueller, J., et al. 2007, ApJ, 654, 1052

Pareschi, G., \& Ferrando, P. 2005, Exper. Astron., 20, 139

Pittard, J. M., \& Dougherty, S. M. 2006, MNRAS, 372, 801

Preibisch, T., Kim, Y.-C., Favata, F., et al. 2005, ApJS, 160, 401

Scalo, J. M. 1986, Fund. Cosmic Phys., 11, 1

Veronig, A., Vršnak, B., Dennis, B. R., et al. 2002, A\&A, 392, 699 